\title{
Impactos no perfil epidemiológico da Dengue em meio a Pandemia da COVID-19 em
}

\author{
Sergipe \\ Impacts on the epidemiological profile of Dengue amid the COVID-19 Pandemic in Sergipe \\ Impactos en el perfil epidemiológico del Dengue en medio de la pandemia COVID-19 en Sergipe
}

Recebido: 01/04/2021 | Revisado: 10/04/2021 | Aceito: 13/04/2021 | Publicado: 23/04/2021

\author{
Crysvânia Santos Nascimento \\ ORCID: https://orcid.org/0000-0001-5760-4911 \\ Universidade Tiradentes, Brasil \\ E-mail: crysvania39@gmail.com \\ João Paulo Siqueira Correia \\ ORCID: https://orcid.org/0000-0002-8214-0853 \\ Universidade Tiradentes, Brasil \\ E-mail: jpsiqueirabrr@gmail.com \\ Camila Conceição Santos Temóteo \\ ORCID: https://orcid.org/0000-0002-6469-2996 \\ Universidade Tiradentes, Brasil \\ E-mail: milamedtemoteo@gmsil.com \\ André Luiz Baião Campos \\ ORCID: https://orcid.org/0000-0002-5794-7196 \\ Universidade Tiradentes, Brasil \\ E-mail: andre.baiao@souunit.com.br
}

\begin{abstract}
Resumo
A dengue é uma patologia viral aguda, sistêmica, única e autolimitada em sua forma clássica, transmitida por artrópodes. Entretanto, o ano de 2020, foi marcado pela pandemia do novo Coronavírus (COVID-19) e, verificou-se uma diminuição nos casos de doenças notificadas causadas por arboviroses quando contrastado com o mesmo período do ano de 2019. O presente trabalho tem como proposta convidar os profissionais de saúde e comunidade em geral à reflexão sobre a importância da notificação busca e assistência a dengue no estado de Sergipe em meio a pandemia do novo coronavírus, a fim de demonstrar os altos níveis de subnotificação e ausência da procura por assistência que podem causar impactos na qualidade de vida da população geral. Trata-se de um estudo observacional transversal, com abordagem analítica quantitativa, com dados coletados de 2019-2020 através da análise de Boletins Epidemiológicos e notificações do SINAN (Sistema de Agravos e Notificações) e SIM (Sistema de Informação de Mortalidade). Os dados obtidos mostraram que houve uma redução, comparando-se os anos 2019-2020, de 5164 casos notificados em 2019 para 959 casos (18,5 \% do primeiro) em 2020, nas notificações nos casos de Dengue no período estudado da semana epidemiológica 12-30. As informações citadas mostraram que, provavelmente, os esforços direcionados a contenção da nova entidade emergente, COVID-19, dificultaram as notificações e os diagnósticos efetivos da patologia citada.
\end{abstract}

Palavras-chave: Dengue; Pandemia 2020; Atenção primária à saúde.

\begin{abstract}
Dengue is an acute, systemic, unique and self-limited viral pathology in its classic form, transmitted by arthropods. However, the year 2020 was marked by the pandemic of the new Coronavirus (COVID-19) and, there was a decrease in the cases of notified diseases caused by arboviruses when contrasted with the same period in 2019 . The present work aims to proposal to invite health professionals and the community in general to reflect on the importance of notification seeking and assisting dengue in the state of Sergipe amid the pandemic of the new coronavirus, in order to demonstrate the high levels of underreporting and absence of the demand for assistance that may impact the quality of life of the general population. This is a cross-sectional observational study, with a quantitative analytical approach, with data collected from 2019-2020 through the analysis of Epidemiological Bulletins and notifications from SINAN (System of Diseases and Notifications) and SIM (Mortality Information System). The data obtained showed that there was a reduction, comparing the years 2019-2020, from 5164 cases notified in 2019 to 959 cases (18.5\% of the first) in 2020, in the notifications in the cases of Dengue in the period studied from epidemiological week 12-30. The aforementioned information showed that, probably, the efforts directed at containing the new emerging entity, COVID-19, hindered the notifications and the effective diagnoses of the aforementioned pathology.
\end{abstract}

Keywords: Dengue; Pandemic 2020; Primary health care. 


\begin{abstract}
Resumen
El dengue es una patología viral aguda, sistémica, única y autolimitada en su forma clásica, transmitida por artrópodos. Sin embargo, el año 2020 estuvo marcado por la pandemia del nuevo Coronavirus (COVID-19) y, hubo una disminución en los casos de enfermedades notificadas por arbovirus cuando se contrasta con el mismo período en 2019. El presente trabajo tiene como objetivo proponer a invitar a los profesionales de la salud y a la comunidad en general a reflexionar sobre la importancia de la búsqueda de notificaciones y la asistencia al dengue en el estado de Sergipe en medio de la pandemia del nuevo coronavirus, con el fin de demostrar los altos niveles de subregistro y la ausencia de demanda de asistencia que puede impactar la calidad de vida de la población en general. Se trata de un estudio observacional transversal, con enfoque analítico cuantitativo, con datos recolectados del 2019-2020 mediante el análisis de Boletines Epidemiológicos y notificaciones del SINAN (Sistema de Enfermedades y Notificaciones) y SIM (Sistema de Información de Mortalidad). Los datos obtenidos mostraron que hubo una reducción, comparando los años 2019-2020, de 5164 casos notificados en 2019 a 959 casos (18,5\% del primero) en 2020, en las notificaciones en los casos de Dengue en el período estudiado desde la semana epidemiológica 12-30. La información mencionada mostró que, probablemente, los esfuerzos dirigidos a contener la nueva entidad emergente, COVID-19, dificultaron las notificaciones y los diagnósticos efectivos de la patología mencionada.
\end{abstract}

Palabras clave: Dengue; Pandemia 2020; Atención primaria de salud.

\title{
1. Introdução
}

A dengue é uma patologia viral aguda, sistêmica, única e autolimitada em sua forma clássica, transmitida por artrópodes (Brasil, 2016). Trata-se de um problema de saúde pública mundial, principalmente nas regiões endêmicas tropicais, devido as condições socioepidemiológicas de propagação do Aedes aegypiti, mosquito vetor de maior importância (Brasil, 2002).

Sua ocorrência no Brasil é de forma continuada, e ainda, em forma de epidemias, principalmente associadas a novos sorotipos para aquela região específica ou alteração do principal sorotipo (Brasil, 2009). Destaca-se ainda, o padrão sazonal da incidência da doença, relacionados com especificidades de cada região, com a população de mosquitos (quantidade, disseminação e distribuição) do gênero Aedes contaminados - até então, a espécie Aedes aegypti é o principal transmissor nas Américas -, da propensão de contaminação individual ou coletiva (imunidade, etnia, patologias anteriores, padrão nutricional) e, como citado, alteração de sorotipos predominantes ou surgimento de novos (Fonseca, 2017).

O diagnóstico é clínico-epidemiológico e laboratorial, tanto usando exames inespecíficos como hemograma e prova do laço, quanto exames específicos de pesquisa de anticorpos e detecção viral (Biassoti \& Ortiz, 2017). O Ministério da saúde, visando evitar a dificuldade do diagnóstico das formas graves e a conduta terapêutica adequada, propõe estadiamento clínico em 4 grupos (A-D), evoluindo na escala de gravidade e necessidade de intervenções, tendo como base terapêutica a hidratação vigorosa e imediata, e medicação antitérmica e analgésica de suporte, reforçando o não uso dos salicilatos ou anti-inflamatórios não esteroides (Brasil, 2016).

Nos últimos 40 anos, mais de 30 patologias infecciosas foram descobertas ou reemergentes com variadas origens etiológicas e causando diversos impactos na saúde, cursando com alterações nos perfis de morbimortalidade a nível global, gerando pandemias (Jimenez, 2013). A OMS declarou pandemia global pela COVID-19 em 11 de março de 2020 e, até o dia 05 de novembro de 2020, havia um total de 47.930 .397 casos confirmados da doença, com 1.221.781 mortes globalmente, afetando 219 países (Who, 2020). A COVID-19 é uma patologia viral causada pelo SARS-CoV-2, de alta transmissibilidade, principalmente pelo contato de gotículas respiratória de indivíduos contaminados sintomáticos. Pode cursar com quadros de leve a grave de Síndrome Respiratória Aguda, como também, outras afecções inespecíficas como febre, tosse, dispneia, mialgia e fadiga, sintomas do trato respiratório superior, sintomas gastrintestinais, alterações cutâneas, dentre outros, entendese que, faltam muitos detalhes a serem elucidados (Brasil, 2020).

No contexto da pandemia de 2020, a OMS declarou critérios clínicos, epidemiológicos e laboratoriais para o diagnóstico da COVID-19, podendo dessa forma, classificar o caso como suspeito, provável e confirmado para proceder nas condutas. É válido salientar que “o julgamento clínico e de saúde pública deve ser usado para determinar a necessidade de 
investigação adicional e pacientes que não atendem estritamente aos critérios clínicos ou epidemiológicos” (Who, 2020).

Com os esforços dos sistemas de saúde e organizações mundiais para conter a disseminação da nova patologia em questão, fica o questionamento quanto a capacidade dos programas de vigilância de doenças emergentes, reemergentes e concomitantes, como é o caso da dengue, podendo impactar gravemente o sistema de saúde pública cursando com desaceleração da saúde (Cardona-ospina et al., 2020).

Enquanto a COVID-19 alarma mundialmente, países sul-americanos, como Brasil, correm um risco muitíssimo elevado de surto de dengue, visto que as taxas de infecção por esse arbovírus cresce alarmantemente. Acredita-se, ainda, que COVID-19 e Dengue estejam ocorrendo simultaneamente nessas regiões, sendo um difícil processo e um desafio preocupante o combate de ambas patologias, isoladamente ou em coinfecção (Miah \& Husna, 2020). A probabilidade de coinfecção, dos dois surtos simultâneos e de colapso dos sistemas de saúde, tanto privados quanto o Sistema Único de Saúde (SUS) público, preocupam as autoridades (Lorenz et al., 2020). A atenção primária à saúde (APS) tem papel primordial no combate de epidemias como a COVID-19 (doença causada pelo novo Coronavírus), a dengue, e ainda, na promoção, acompanhamento e prevenção à saúde, garantindo acesso, longitudinalidade, integralidade e coordenação do cuidado (Soeiro et al., 2020). Entretanto, acredita-se que haja receio da população na busca de assistência médica por medo do risco de contaminação pela COVID-19.

Um estudo feito por Nacher e colaboradores, em 2020 na Guiana Francesa, mostrou que houve redução no número de atendimentos, tanto porque os casos não urgentes foram postergados, quanto por esse provável pânico de contaminação da população. Além disso, devido ao quadro clínico semelhante, os pacientes eram testados primariamente para COVID-19 e após negativação da amostra, seriam pesquisadas outras causas para o quadro, resultando em atrasos diagnósticos dos casos de Dengue (Nacher et al., 2020).

O objetivo desse estudo está em evidenciar dados que ratifiquem a subnotificação dos casos de Dengue no ano de 2020, devido aos esforços voltados para a pandemia do novo Coronavírus, à subnotificação dos casos, à ausência de busca por assistência médica e receio da população. Para que, dessa forma, haja uma alteração nas estratégias de busca e continuidade do cuidado à população exposta, atualmente e em eventos futuros, a fim de favorecer um melhor atendimento, visando prevenção e promoção em saúde e, ainda, evitar superlotação dos sistemas. Garantindo os princípios de integralidade, universalidade e equidade do Sistema Único de Saúde.

\section{Metodologia}

Trata-se de um estudo observacional, transversal, com abordagem analítica quantitativa, com dados coletados no período de 2019 a 2020, nas semanas epidemiológicas de 12 a 30. Os dados foram obtidos através de revisão de boletins epidemiológicos do Ministério da Saúde, dados do Sistema de Informação de Agravo de Notificação (SINAN) e do Sistema de Informação sobre Mortalidade (SIM) ambos disponibilizados pelo Departamento de Informática do Sistema Único de Saúde do Brasil (DATASUS) através da plataforma Informações de Saúde (TABNET).

Compreende-se que o estudo analítico quantitativo se baseia na coleta de dados numéricos que promovem resultados em grupos que podem ser avaliados comparando suas respectivas grandezas. Dessa forma, utiliza técnicas matemáticas por meio de porcentagens, probabilidades e estatísticas, possibilitando realizar antevisão do evento estudado. (Pereira et al.,2018). Portanto, foi o método de escolha neste trabalho.

Visando aprofundamento teórico foram pesquisados os descritores: dengue, pandemia 2020, atenção primária a saúde, nas bases de dados da Biblioteca Virtual em Saúde (BVS) e Medical Literature Analysis and Retrievel System Online (Medline). Ademais, foi inserido na pesquisa as Diretrizes Nacionais para a Prevenção e Controle de Epidemias de Dengue. Os dados coletados estão disponíveis na rede de internet, cedidos pelo Ministério da Saúde para livre consulta, conferindo domínio 
público e garantindo o sigilo dos seres humanos envolvidos, não necessita, portanto, da submissão ao Comitê de Ética em Pesquisa. A população do estudo foi constituída por sergipanos que morreram ou procuraram atendimento referente a suspeita de Dengue no período de 2019-2020.

\section{Resultados e Discussão}

A pandemia pela COVID-19 desestruturou todo um sistema de saúde mundial e o organizou para contenção da nova entidade emergente, no estado de Sergipe não foi diferente. O Gráfico 1 mostra a descrição da incidência da Dengue nas semanas epidemiológicas 8 a 30 do ano de 2019 e 2020.

Gráfico 1. Incidência da Dengue 2019-2020.

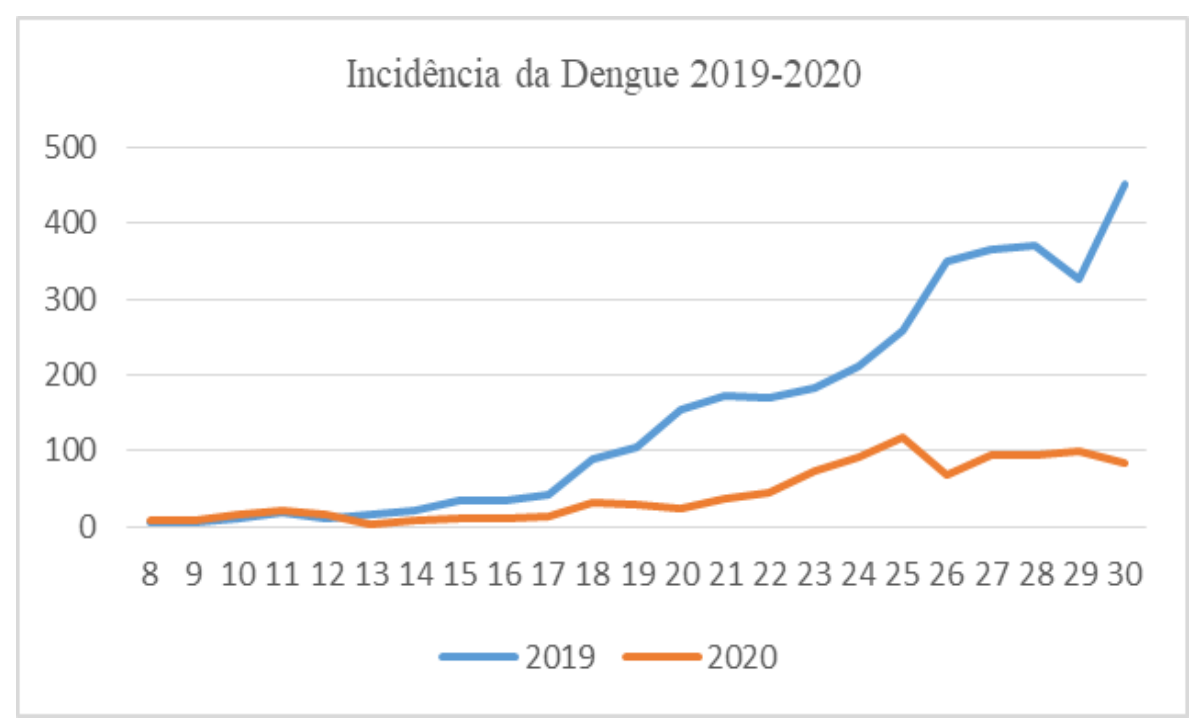

Fonte: Autores, com base em dados do SINAN.

Os dados evidenciados revelam uma diminuição da incidência dos casos de Dengue após a semana epidemiológica 12, data esta que marca o início dos casos notificado de COVID-19. É observado ainda, que, nesse mesmo período em anos anteriores, exemplificado pelo ano de 2019, e evidenciado pela revisão de literatura neste trabalho, a Dengue teria aumento do número de indivíduos acometidos, o que não foi obtido no ano de 2020. Na capital sergipana, no ano de 2020 foram confirmados apenas 912 casos, em 2019 esse número chegou a 3442. Os óbitos por Dengue no estado em 2019 foram 13, enquanto no ano da pandemia emergente não houve notificação de nenhum óbito tendo a Dengue como causa.

Esses dados ressaltam três grandes questões: quanto a subnotificação dos casos, quanto ao receio da população na busca por auxílio médico e possível contaminação pelo novo agente, e quanto a coinfecção Dengue e Covid-19. Devido aos esforços voltados para a contenção do novo agente infeccioso e, inclusive, sintomatologia semelhante e convergente entre as patologias estudadas, fica difícil para o profissional de saúde fazer o diagnóstico diferencial, sendo que, no cenário de pandemia mundial, todo caso de síndrome gripal era considerado COVID-19 e primariamente testado para o mesmo e então, se negativado, iria em busca de outras causas para o quadro, resultando em atrasos diagnósticos dos casos de Dengue. Lembrando que, a redução no número de atendimentos ocorreu também porque os casos não urgentes foram postergados, e, ainda, por esse provável pânico de contaminação da população já citado. Quando se observa os casos notificados, compreendemos que a busca por auxílio médico também, e a hipótese subnotificação dos casos se consolida.

O Gráfico 2 mostra a incidência de Dengue nas semanas epidemiológicas (SE) 12-30 do ano de 2019-20 e COVID nas SE $12-30$ de 2020. 
Gráfico 2. Incidência de Dengue x COVID.

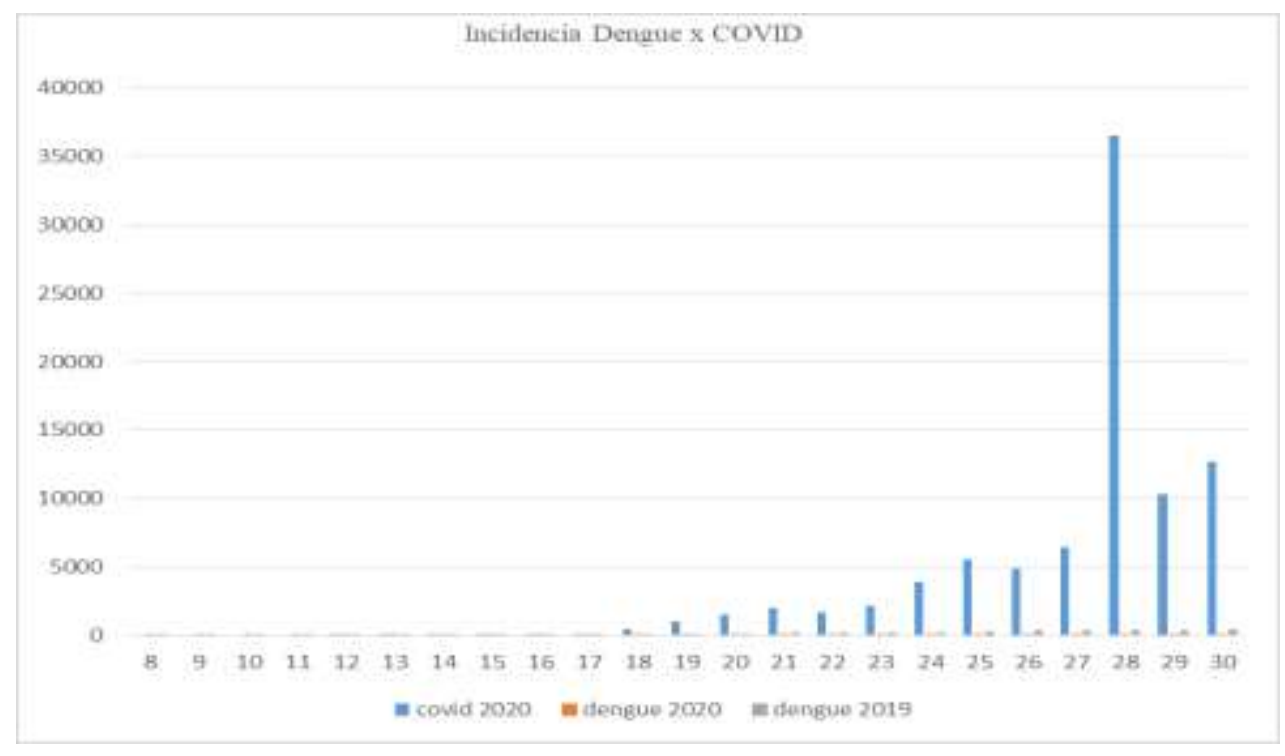

Fonte: Autores, com base em dados do SINAN.

Ainda que poucos casos de Dengue em comparação com a extensão dos casos de COVID, nota-se que no ano de 2019 os números ainda eram consideráveis, e no ano de 2020 a COVID-19 ascendeu exponencialmente nos casos notificados, enquanto a dengue obteve queda.

Quando comparamos com os casos de Chikungunya, outra arbovirose relacionada ao Aedes aegypti, no ano de 2019 tivemos apenas 708 casos e em 2020, surpreendentemente, 3820. Esse dado remete muitas dúvidas quanto a subnotificação dos casos nos anos anteriores ou, eventualmente, diminuição das medidas de controle do vetor ocasionando aumento dos casos da doença no referido ano. Compreende-se que, os esforços voltados para pandemia impossibilitaram os agentes de endemias de realizarem as fiscalizações dos criadouros do mosquito nas casas da população. Logo, essa estatística também remete que os casos de Dengue deveriam ter aumentado, corroborando com a hipótese de subnotificação dos casos. Acredita-se que a notificação dos casos de Chikungunya tenha sido feita especialmente devido ao seu quadro de artralgia intensa, o que faz o paciente procurar auxílio médico.

De acordo com os dados encontrados no presente estudo, houve uma redução, comparando-se os anos 2019-2020, de 5164 casos notificados em 2019 para 959 casos (18,5 \% do primeiro) em 2020, nas notificações nos casos de Dengue no período estudado da semana epidemiológica 12-30. Os dados obtidos mostraram que, provavelmente, os esforços direcionados a contenção da nova entidade emergente, COVID-19, inviabilizaram as notificações e os diagnósticos efetivos da patologia citada. Além disso, a sintomatologia semelhante entre as doenças pode ter contribuído, e ainda, casos de coinfecção, dessa forma, acredita-se que, como ambas não tem um tratamento específico e tendem a autoressolução, não foi realizada a testagem diagnóstica para dengue e, portanto, não foi feita a notificação. Quanto à busca por assistência médica específica, entende-se que o pânico da população de contaminação pela COVID-19 dificultou a notificação e retardaram os diagnósticos, já que, não procuraram auxílio, ou procuraram com uma frequência menor.

\section{Conclusão}

O tema do presente estudo levanta questões que podem ser ampliadas e aprofundadas por novos estudos de forma a compreender melhor e refletir sobre os dados aqui levantados. Dessa forma, pode-se estabelecer a importância de protocolos, principalmente em áreas endêmicas, para que as notificações sejam efetivas e medidas de contenção da doença sejam mais 
rigorosas. Tendo em vista que a Dengue, embora tenha evolução benigna na maioria dos casos, tem os quadros com distúrbios hemorrágicos, e estes, são fatais, torna-se crucial o desenvolvimento de políticas de incentivo à notificação, contenção do agente, educação populacional e medidas de higiene sanitária, já que, o diagnóstico e monitorização precoce, são indispensáveis e ditadores prognósticos.

Para trabalhos futuros, sugere-se uma investigação aprofundada quanto aos protocolos seguidos pelas instituições no manejo da dengue e da COVID-19, assim como um melhor detalhamento das causas da diminuição das notificações de casos suspeitos e confirmados.

\section{Referências}

Aracaju/SMS (2020). Todos contra o corona. https://todoscontraocorona.net.br/boletins-covid/page/18/

Biassoti, A. V. I., \& Ortiz, M. A. L. (2017); Diagnóstico laboratorial da dengue. Revista Uningá Review. 29, 3-4.

Cardona-ospina, J. A., et al. (2020) Dengue and COVID-19, overlapping epidemics? An analysis from Colombia. Journal of Medical Virology.

Chen N. et al. (2020). Características epidemiológicas e clínicas de 99 casos de novos casos de pneumonia por coronavírus em Wuhan, China: um estudo descritivo. Lanceta. $395:$ :507-513.

Datasus. (2021) Sistema de Informação de Agravo de Notificação. http://tabnet.datasus.gov.br/cgi/tabcgi.exe?sinannet/cnv/denguebse.def.

Fonseca, B., \& Abrão, E. (2020). Dengue. In: Salomão, Reinaldo. Infectologia: Bases clínicas e tratamento / Reinaldo Salomão 55, $452-456$.

Jimenez, L. C. V. (2020). Epidemias y pandemias: una realidade para el siglo XXI. Un mundo y una salud. Rev. Lasallista Investig. 10(10, 7-8.

Lorenz, C., Azevedo T. S., \& Chiaravalloti-Neto F. (2020). COVID-19 and dengue fever: a dangerous combination for the health system in Brazil. Travel Med Infect Dis.

Mascarenhas, M. D. M., et al. (2020). Ocorrência simultânea de COVID-19 e dengue: o que os dados revelam? Cadernos de Saúde Pública. 36.

Miah, M. D. A., \& Husna, A. (2020). Coinfection, coepidemics of COVID-19, and dengue in dengue-endemic countries: A serious health concern. Journal of Medical Virology.

Ministério da Saúde (2002). Secretaria de Vigilância em Saúde. Departamento de Vigilância Epidemiológica. Programa Nacional de Controle da Dengue. Brasília: Fundação Nacional de Saúde.

Ministério da Saúde (2009). Secretaria de Vigilância em Saúde. Departamento de Vigilância Epidemiológica. Diretrizes nacionais para a prevenção e controle de epidemias de dengue. Brasília: MS. Série A. Normas e Manuais Técnicos.

Ministério da Saúde (2016). Secretaria de Vigilância em Saúde. Dengue diagnóstico e manejo clínico. Série A. Normas e Manuais Técnicos. (5a ed.), DF.

Ministério da Saúde (2020) Secretaria de Atenção Primária à Saúde. Protocolo de manejo clínico do coronavírus (covid-19) na atenção primária à saúde. Versão 9. Brasília, DF.

Nacher, M., et al. (2020). Simultaneous dengue and COVID-19 epidemics: Difficult days ahead? PLOS Neglected Tropical Diseases.

Opas/oms | Organização Pan-Americana da Saúde. (2020). Controle do Aedes aegypti em cenário de transmissão simultânea de COVID-19. https://www.paho.org/pt/documentos/control-aedes-aegypti-escenario-transmision-simultanea-covid-19.

Pereira A. S. et al. (2018). Metodologia da pesquisa científica. [free e-book]. Santa Maria/RS. Ed. UAB/NTE/UFSM.

Soeiro, et al (2020). Atenção Primária à Saúde e a pandemia de COVID-19: reflexão para a prática. InterAmerican Journal of Medicine and Health.

Who (World Health Organization). (2020) Coronavirus disease (COVID-19). https://www.who.int/emergencies/diseases/novel-coronavi rus-2019.

Who/oms. (2020). Um guia de orientação da OMS sobre COVID-19. https://www.who.int/news-room/feature-stories/detail/a-guide-to-who-s-guidance.

Yan, G., et al. (2020) Covert COVID-19 and false-positive dengue serology in Singapore. Lancet Infect Dis. 